\title{
Multiple and metachronous esophageal intramural metastases from a gastric adenocarcinoma
}

\author{
Osamu Ikeda ${ }^{1}$, Yasushi Toh ${ }^{1}$, Yoshiro Aoki ${ }^{1}$, Norifumi Harimoto ${ }^{1}$, Jyunya TaOmoto ${ }^{1}$, TakaAki Masuda $^{1}$, \\ Takefumi Ohga ${ }^{1}$, Eisuke Adachi ${ }^{1}$, Yoshinisa Sakaguchi ${ }^{1}$, Takeshi Okamura ${ }^{1}$, Minako Hirahashi ${ }^{2}$, \\ Kenichi NishiYama ${ }^{2}$, and Hideo BabA ${ }^{3}$ \\ ${ }^{1}$ Department of Gastroenterological Surgery, National Kyushu Cancer Center, 3-1-1 Notame, Minami-ku, Fukuoka 811-1395, Japan \\ ${ }^{2}$ Department of Pathology, National Kyushu Cancer Center, Fukuoka, Japan \\ ${ }^{3}$ Department of Gastroenterological Surgery, Graduate School of Medical Sciences, Kumamoto University, Kumamoto, Japan
}

\begin{abstract}
Esophageal squamous cell carcinoma is often accompanied by intramural metastases, and it has been reported to carry a poor prognosis. Intramural metastasis from gastric cancer to the esophageal wall, however, has rarely been reported. We herein report a rare case of a 46-year-old man with an elevated esophageal lesion, resembling a 0-IIa-type esophageal cancer, which was discovered 13 months after a total gastrectomy performed for gastric cancer. The esophageal tumor, resected by endoscopic mucosal resection (EMR), was an adenocarcinoma with the same histology as the previously resected primary gastric cancer, and it showed massive lymphatic permeation. Soon after the EMR, other similar lesions emerged on the esophageal wall. We therefore considered the esophageal tumor to be a systemic expansion of the primary gastric cancer, and we administered the anticancer drug, S-1. Esophageal intramural metastases from a gastric cancer imply a systemic expansion of the gastric cancer, and portend a poor prognosis.
\end{abstract}

Key words Intramural metastasis - Esophageal neoplasm · Gastric cancer $\cdot$ S-1

\section{Introduction}

Esophageal squamous cell carcinoma is often accompanied by intramural metastasis, which predicts a poor prognosis. The intramural metastasis of a gastric cancer to the esophageal wall, however, has rarely been reported. We herein report the case of a recurrent gastric adenocarcinoma with intramural metastases to the esophagus occurring 13 months after a total gastrectomy with lymph node dissection.

Offprint requests to: $\mathrm{Y}$. Toh

Received: May 31, 2007 / Accepted: December 16, 2007

\section{Case report}

A 46-year-old man was admitted to the Department of Gastroenterological Surgery, National Kyushu Cancer Center, because of gastric cancer of the cardia. A barium meal examination and upper gastrointestinal endoscopy disclosed a Borrmann type II tumor with esophageal invasion. Biopsy specimens from the tumor demonstrated moderately differentiated adenocarcinoma.

The patient underwent a total gastrectomy and lymph node dissection, with a combined resection of the spleen and the gallbladder. The resected specimen showed a tumor of the cardia, measuring $5 \mathrm{~cm} \times 4 \mathrm{~cm}$, which had macroscopically infiltrated the entire thickness of the gastric wall, and which had invaded the abdominal esophagus to within $1 \mathrm{~cm}$ of the esophagogastric junction (Fig. 1A). The tumor of the cardia was histologically revealed to be a moderately differentiated adenocarcinoma which had marked lymphatic permeation in the submucosal layer of the esophagogastric junction (Fig. 1B). The proximal margin of the resected specimen was free of residual cancer cells and free of lymphatic invasion. Metastatic lymph nodes were found in some of the paracardial and the lesser curvature regions. The TNM stage of the tumor was $\mathrm{pT} 3 \mathrm{pN} 2 \mathrm{M} 0$.

Postoperatively, the patient received no adjuvant chemotherapy. However, 13 months after the operation, upper gastrointestinal endoscopy disclosed a flatsurfaced, somewhat elevated tumor in the esophagus, $32 \mathrm{~cm}$ from the incisor teeth, which macroscopically resembled a 0-IIa-type primary esophageal cancer (Fig. 2A). This lesion measured $7 \mathrm{~mm}$ in diameter and was unstained with Lugol's solution (Fig. 2B). The tumor was $5 \mathrm{~cm}$ proximal to the esophago-jejunal anastomosis, which was free of recurrent tumor. The biopsy specimens indicated a moderately to poorly differentiated adenocarcinoma. We first suspected a primary esophageal cancer, along with the possibility of esophageal intramural metastasis from gastric cancer. Because 


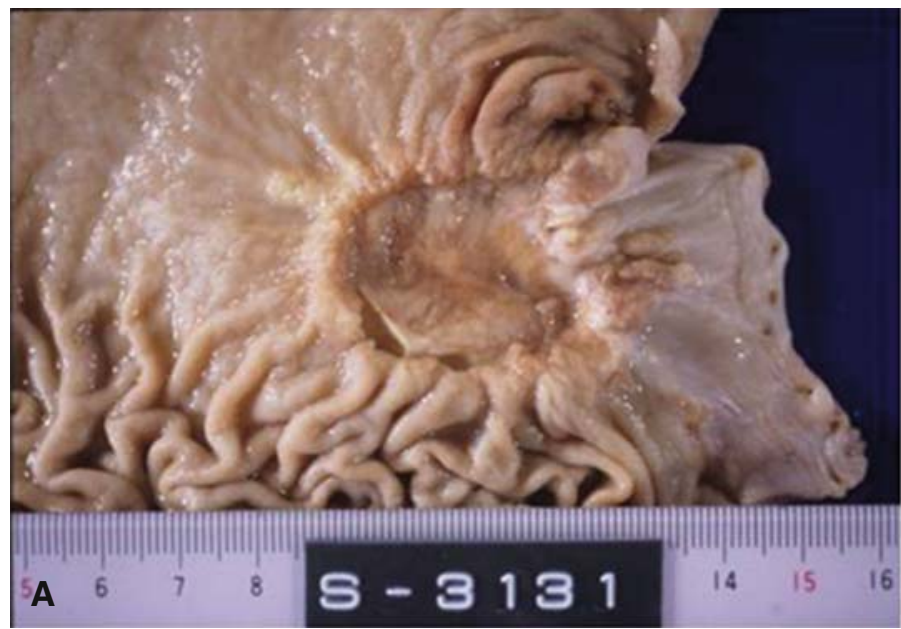

Fig. 1. A Gross appearance of the resected specimen. An ulcerated gastric carcinoma was located in the cardia of the stomach and was found to have invaded to the abdominal esophagus. B Histology of the primary tumor of the cardia

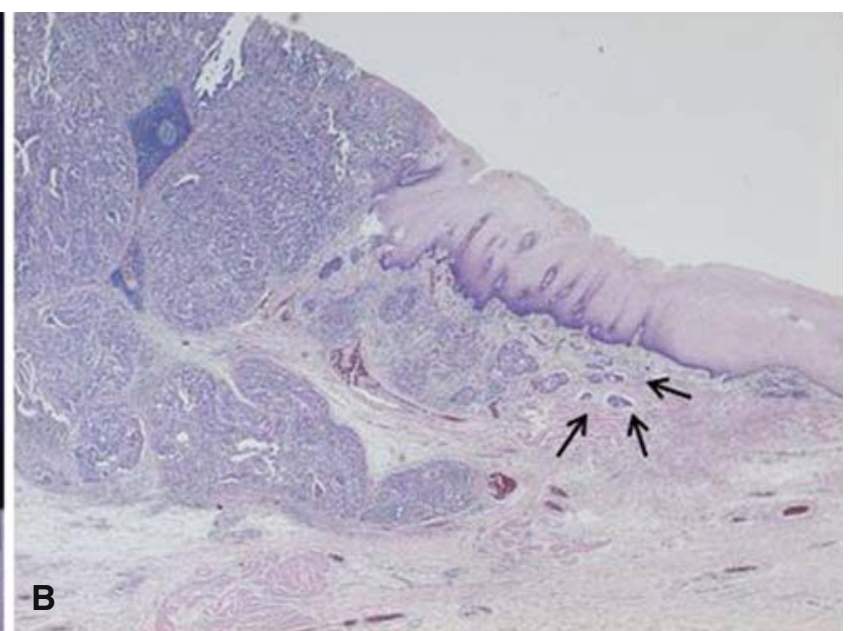

was moderately differentiated adenocarcinoma. Marked lymphatic permeation was seen in the submucosal layer of the esophagogastric junction (arrows). $\mathrm{H} \& \mathrm{E}$
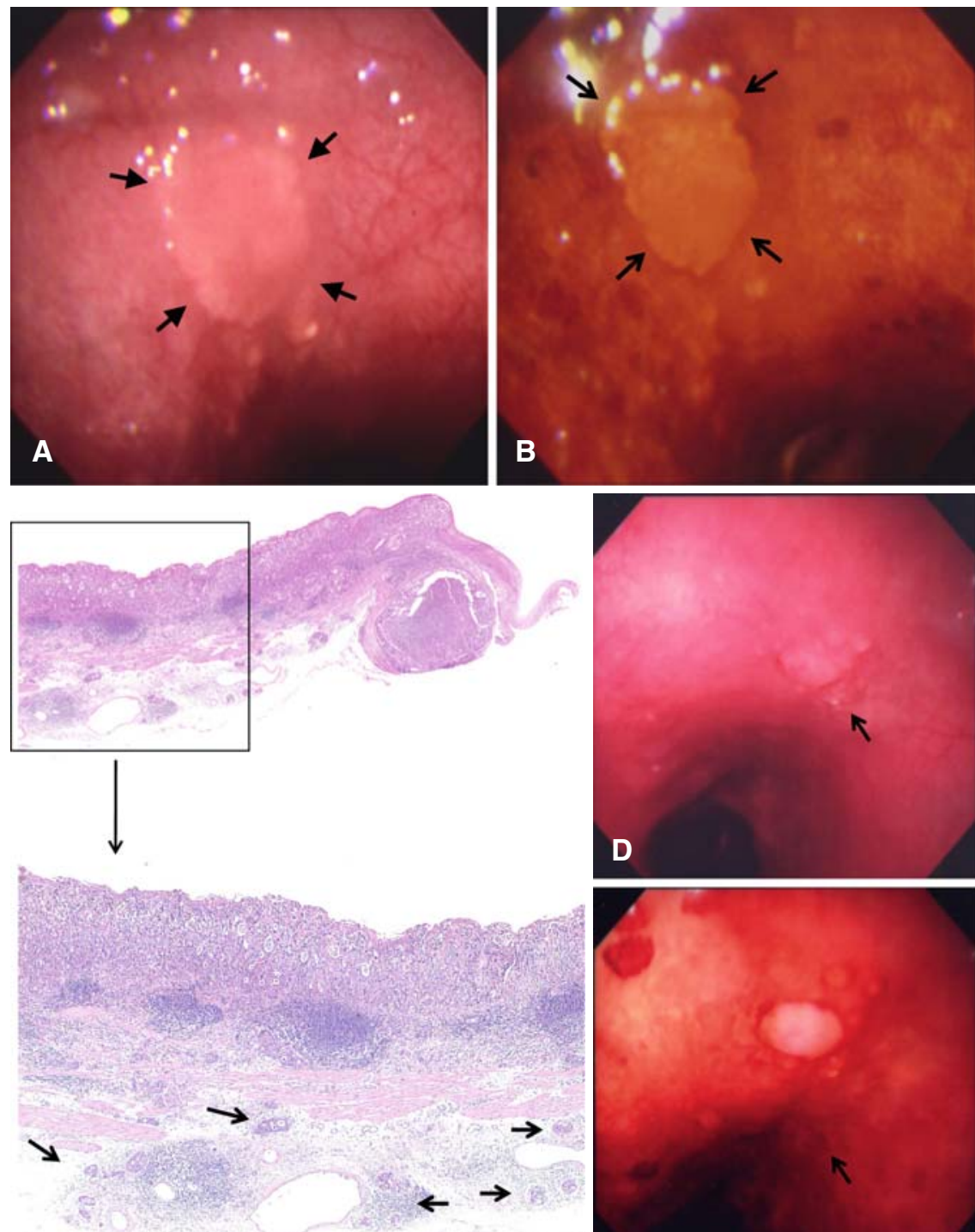

C
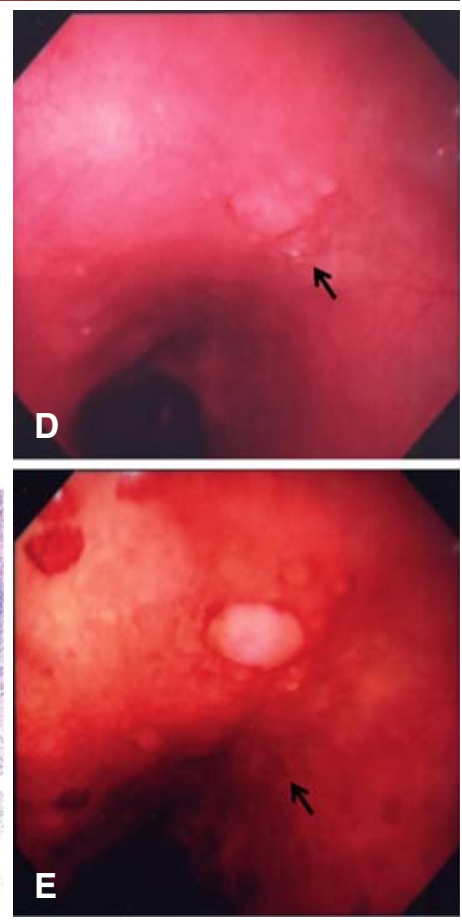

Fig. 2. A Endoscopic view of a flat elevation of the esophagus which was located $5 \mathrm{~cm}$ on the oral side of the esophagojejunal anastomosis (arrows). B The elevation was unstained by the application of Lugol's solution. C Histological findings of endoscopic mucosal resection (EMR) specimen. Moderately to poorly differentiated adenocarcinoma is invading the submucosal layer of the esophageal wall, with massive lymphatic permeation (arrows in inset). H\&E. D Upper gastrointestinal endoscopy disclosed other lesions, similar to that resected by the EMR, in the thoracic esophagus (arrow). E These elevations were also unstained by the application of Lugol's solution (arrow) 
esophageal endoscopic ultrasound $(30 \mathrm{MHz})$ revealed that the extent of the tumor invasion was limited to the proper mucosal layer, the patient underwent a cap-assisted endoscopic mucosal resection (EMR). The tumor resected by the EMR demonstrated massive lymphatic permeation and displayed the same histological appearance as the previously resected gastric cancer (Fig. 2C), therefore, we interpreted this esophageal tumor as an intramural metastasis from the gastric cancer, having spread via the lymphatic vessels in the esophageal wall. Two weeks after the EMR, several tiny flat nodules, similar to the previous lesion, appeared $3 \mathrm{~cm}$ proximal to the EMR scar, and were shown to be moderately to poorly differentiated adenocarcinoma by histological examination. Because these new lesions were also thought to be intramural metastases from the gastric cancer, the administration of an oral fluoropyrimidine, S-1 (100 mg/body), was started. However, similar flat nodules of various sizes developed throughout the thoracic esophagus (Fig. 2D,E). The patient developed multiple lymph node and bone metastases 23 months after the first recurrence, and unfortunately died 27 months after the first recurrence.

\section{Discussion}

In Japan, squamous cell carcinoma is the most frequently identified histological type of primary esophageal cancer, occurring in $92.0 \%$ of cases.Adenocarcinoma is found in only $1.6 \%$ of cases [1]. In esophageal squamous cell carcinoma, intramural metastases are reported to occur with an incidence ranging from $11.9 \%$ to $16.7 \%$ [2-4], and are considered to be one of the most important prognostic factors [4]. An intramural metastasis of a gastric adenocarcinoma to the esophagus, however, has rarely been reported. The first case of such a metastasis was reported in the English-language literature in 1998 [5], and to our knowledge, only seven cases have since been reported, apart from the present case [5,6]. Furthermore, only ten cases of such metastasis have been reported in the Japanese literature, even though Japan is a country with a very high incidence of gastric cancer [7]. Therefore, the occurrence of intramural metastasis of gastric cancer to the esophagus is thought to be a rare event.

Szántó et al. [6] documented the finding that intramural esophageal metastases were verified in $6(4.19 \%)$ of 143 patients with cardial adenocarcinoma in a retrospective study.

In all seven cases reported in the English-language literature [5,6], the intramural esophageal metastases were found synchronously along with the primary gastric cancer. Furthermore, six of the seven patients died within 1 year of diagnosis of the metastasis. However, the present patient had metachronous metastases and survived for 27 months after the first recurrence, having been treated with S-1.

It was difficult for us to be certain as to the source of the metastases in our patient. However, both the abundant lymphatic network within the esophageal wall and the marked lymphatic invasion of the primary gastric cancer led us to believe that the gastric cancer cells had invaded the lymphatic vessels and spread into the esophageal wall. Hirota et al. [5] and Szántó et al. [6] noted that esophageal intramural metastases, both from gastric adenocarcinoma and from esophageal squamous cell carcinoma, had the same clinical impact; the intramural metastases from these cancers indicated systemic expansion of cancer through the intramural lymphatic system and heralded a poor prognosis [6]. In our patient, because it was possible that the solitary esophageal flat elevation may have been a primary esophageal cancer, judging from its macroscopic appearance, we first resected it by EMR. However, the histological examination strongly supported the possibility that this elevated lesion was an intramural metastasis from the gastric cancer. Moreover, immediately after the resection, new similar lesions emerged on the oral side of the esophagus. The EMR specimen showed a 0-IIa-type tumor located within the mucosal layer, with lymphatic invasion. This histological finding may indicate that an 0-IIa-type tumor is a primary esophageal adenocarcinoma. Nishimaki et al. [2] defined the morphological features of intramural metastases from esophageal carcinoma as the gross appearance of a submucosal tumor without any intraepithelial cancer extension. However, the following findings strongly led us to believe that the tumor in our patient was indeed an intramural metastasis: (1) the finding of massive lymphatic invasion both in the previous gastric cancer and in the tumor resected by EMR; (2) the finding of the same histological type in both tumors; (3) the emergence of multiple esophageal lesions soon after the EMR resection; and (4) the very low incidence of esophageal adenocarcinoma in Japan.

We considered the state of the disease in our patient to be a systemic expansion of the gastric adenocarcinoma and we administered S-1 instead of performing a further EMR or an esophagectomy. The patient survived for longer than almost all other such patients who were treated with an esophagectomy [5,6]. Some recent studies have reported the effectiveness of systemic chemotherapy, with agents such as $\mathrm{S}-1$, for the treatment of recurrent gastric cancers [8,9]. From the viewpoint of systemic disease, the administration of systemic chemotherapy, rather than performing an esophagectomy, may improve the prognosis of patients with esophageal intramural metastasis from gastric cancer. 
In conclusion, the occurrence of esophageal intramural metastases from gastric cancer implies a systemic expansion of the gastric cancer, and portends a poor prognosis, in the same manner as that observed for intramural metastases from esophageal cancer. Determining the optimal treatment for such metastases requires the further accumulation of data regarding esophageal intramural metastases.

\section{References}

1. The Japanese Society for Esophageal Diseases. Comprehensive registry of esophageal cancer in Japan $(1998,1999)$ and long-term results of esophagectomy in Japan (1988-1997). 3rd ed. Chiba: The Japanese Society for Esophageal Diseases; 2002.

2. Nishimaki T, Suzuki T, Tanaka Y, Aizawa K, Hatakeyama K, Muto T. Intramural metastases from thoracic esophageal cancer: local indicators of advanced disease. World J Surg 1996;20:32-7.

3. Takubo K, Sasajima K, Yamashita K, Tanaka Y, Fujita K. Prognostic significance of intramural metastasis in patients with esophageal carcinoma. Cancer 1990;65:1816-9.
4. Kuwano H, Watanabe M, Sadanaga N, Kamakura T, Nozoe T, Yasuda M, et al. Univariate and multivariate analyses of the prognostic significance of discontinuous intramural metastasis in patients with esophageal cancer. J Surg Oncol 1994;57:17-21.

5. Hirota T, Nishimaki T, Suzuki T, Komukai S, Kuwabara S, Aizawa $\mathrm{K}$, et al. Esophageal intramural metastasis from an adenocarcinoma of the gastric cardia: report of a case. Surg Today 1998; 28:1160-2.

6. Szántó I, Voros A, Nagy P, Gonda G, Gamal EM, Altorjay A, et al. Esophageal intramural metastasis from adenocarcinoma of the gastroesophageal junction. Endoscopy 2002;34:418-20.

7. Singh K, Ghoshal UC. Causal role of Helicobacter pylori infection in gastric cancer: an Asian enigma. World J Gastroenterol 2006; 12:1346-51.

8. Takahashi I, Kakeji Y, Emi Y, Sakurai M, Yonemura Y, Kimura Y, et al. S-1 in the treatment of advanced and recurrent gastric cancer: current state and future prospects. Gastric Cancer 2003; 6 Suppl 1:28-33.

9. Cho H, Konishi K, Tsuburaya A, Kobayashi O, Sairenji M, Motohashi $\mathrm{H}$, et al. Longterm control of advanced and recurrent gastric cancer (ARGC) by S-1. Gastric Cancer 2003;6 Suppl 1:24-7. 